\title{
Fast Robust Gate-Drivers with Easily Adjustable Voltage Ranges for Driving Normally-On Wide-Bandgap Power Transistors
}

\author{
Pieter Jacqmaer*, Jordi Everts ${ }^{\dagger} \|$, Ratmir Gelagaev ${ }^{\ddagger}$, Peter Tant ${ }^{\S}$, Johan Driesen $\llbracket$ \\ ${ }^{*}$ K.U.Leuven/Department of Electrical Engineering (ESAT), Heverlee, Belgium, e-mail: pieter.jacqmaer@gmail.com \\ ${ }^{\dagger}$ K.U.Leuven/Department of Electrical Engineering (ESAT), Heverlee, Belgium \\ (and affiliated with KHLim, Diepenbeek, Belgium), e-mail: jordi.everts@esat.kuleuven.be \\ ${ }^{\ddagger}$ K.U.Leuven/Department of Electrical Engineering (ESAT), Heverlee, Belgium, e-mail: ratmir.gelagaev@esat.kuleuven.be

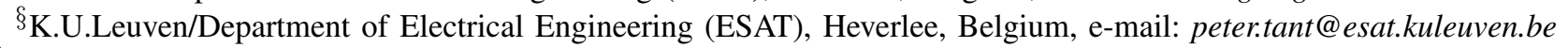 \\ IK.U.Leuven/Department of Electrical Engineering (ESAT), Heverlee, Belgium, e-mail: johan.driesen@esat.kuleuven.be
}

\begin{abstract}
Wide-bandgap (WBG) semiconductors, such as gallium nitride (GaN), are more and more being used in switching power devices. An AlGaN/GaN/AIGaN Double Heterojunction Field Effect transistor (DHFET) was developed in previous work and needed to be tested. The used test circuit was a buck converter. This type of converter, in addition with the normally-on switching behaviour of the GaN-based transistors, requires dedicated gate drive circuitry, resulting in the development of three types of gatedrivers. This paper presents the topology and performance of these drivers. Because of the type of converter, the drivers need to be galvanically isolated. Furthermore, because the experimental GaN transistors are normally-on, the drivers need to be robust so that they apply a negative gate-to-source voltage to switch off the transistor in case an error occurs in the driver. A third requirement for the drivers is that it has to be easy to adjust the voltage levels, in order to test the devices at different gate-to-source voltage conditions. A final requirement is that it has to be possible to construct the drivers with readily available electronic components. Because the drivers are galvanically isolated, there is a parasitic isolation capacitance in the DC-DC-converter of the drivers. This gives rise to a common-mode current which possibly can disturb the operation of the driver. The article also discusses this common-mode problem.
\end{abstract}

Index Terms-Gate-driver, isolated driver, normally-on transistor, wide-bandgap semiconductor

\section{INTRODUCTION}

Recently, wide-bandgap (WBG) semiconductors are more and more being used in switching power devices. The reason for this evolution is that, because of the higher bandgap, WBG-materials can withstand a higher reverse voltage for the same layer thickness in comparison with silicon. Hence, for the same voltage rating, devices can be downscaled and thus, the length of the channel and also the gate-drain capacitance in Field Effect Transistors (FETs) decrease. This, and the fact that the saturated drift velocity is more than twice than that of silicon [1], results in faster switching frequencies for the WBG-devices.

1|| This research is partly funded by a Ph.D grant of the Institute for the Promotion of Innovation through Science and Technology in Flanders (IWT-Vlaanderen). Jordi Everts is a doctoral research assistant of IWT-Vlaanderen.
A wide-bandgap semiconductor is a material having a bandgap higher than $2 \mathrm{eV}$ [2], being almost twice as high as that of silicon (1.12 eV [1] [2]). Silicon carbide $(\mathrm{SiC})$ and gallium nitride $(\mathrm{GaN})$ are examples of WBG-materials, offering bandgaps of $3.03 \mathrm{eV}(6 \mathrm{H}-$ $\mathrm{SiC}), 3.26 \mathrm{eV}$ (4H-SiC), and $3.45 \mathrm{eV}(\mathrm{GaN})$ [1] [2] respectively. An AlGaN/GaN/AlGaN Double Heterojunction Field Effect Transistor (DHFET) was developed [3] and some important characteristics of the device needed to be measured in order to be able to compare their performance with competitor devices of other research institutions. For instance, both the static and dynamic onresistances and also the gate charges had to be measured. A buck (step-down) converter with an input voltage of $400 \mathrm{~V}$, and dimensioned for a current of $20 \mathrm{~A}$, was used as a test circuit. Because the potential of the source of the switch in this type of converter varies, an isolated gatedriver is required. The aim of this work is to build fast, dedicated gate-drive circuitry for driving the DHFETs in the test setup of a buck converter.

One of the shortcomings in the existing literature about gate-drivers is that it is not mentioned how the electrical isolation of the gate driver is realized. Neither is it clear how the high and low level gate-to-source voltages are generated. This work wants to give more insight in the practical development of a gate-driver. Furthermore, the GaN-device of our concern is a 'normally-on' transistor, as is the case in many experimental devices. Therefore, the driver needs to be robust and has to switch off the device immediately if the driver fails by applying a negative gate-to-source voltage. Also, the GaN-transistor is constantly under development and it has to be possible to change the levels of the applied gate-to-source voltage. The reason for this is that when a new transistor is developed which is able to work with other gate-to-source voltage levels, the same driver can be used, but only small changes have to be made to components of the driver. Finally, another requirement for the driver is that it must be possible to construct it with readily available electronic components. 
Three types of gate-drivers are developed, which are discussed in the next three sections. In every section, the topology is discussed and the performance of the drivers is assessed. Very often, the performance of a gate-driver is measured by its power consumption [6], [7], [9], [10], [11]. In this article, the performance of the proposed drivers is measured by their maximal switching frequency as well as the fall-time of the gate-to-source voltage waveform by applying them to a range of MOSFETs with a different input-capacitance $C_{i s s}$, and for different drainto-source voltages $V_{d s}$. In the subsequent section, the possible problem of the presence of a parasitic isolation capacitance in the DC-DC-converter of the gate-driver, is discussed, with measurements and simulation results. It is seen that the common-mode current due to this capacitance is of no concern for the operation of the driver. The last section concludes the article.

Many high frequency gate-drivers have been developed recently. Several of them are resonant drivers [4], [5], [6], [7], using a resonant circuit to reduce the gate-drive losses [8]. Due to the gate input capacitance of the transistor to be driven, there is an inherent gate-drive loss when hardswitching is used, which is, for MOSFETs, given by:

$$
P_{\text {loss }}=C_{i s s} \cdot V_{d s}^{2} \cdot f_{s}=Q_{g} \cdot V_{d s} \cdot f_{s}
$$

Here, $C_{i s s}$ is the input capacitance of the MOSFET, $Q_{g}$ is the gate charge, $V_{d s}$ is the drain-to-source voltage and $f_{s}$ is the switching frequency. If the frequency is high enough, these gate-drive losses can offset the advantages gained by other energy savings elsewhere in the circuit. The main goal of resonant gate-drivers is to recover the energy stored in the gate capacitor, so as to reduce the gate-drive losses. This recovered energy is circulating in a resonant tank every switching cycle, instead of being dissipated in the gate resistor and gate-driver during the charge and discharge periods. To build a resonant gatedriver, it usually suffices to add a resonant tank to the topology of an existing hard-switched gate-driver [4]. The drivers of this article can be made resonant by adding such an extra resonant circuit.

\section{FIRST PROPOSED GATE-DRIVER}

\section{A. Topology}

The first gate driver is presented in Fig. 1. In the center, there is an isolated DC-DC-power converter. Because of the requirement that standard, readily available electronic components have to be applied, the NMH0515DCconverter is used. This converter transforms the $5 \mathrm{~V}$ input voltage into $-15 \mathrm{~V}$ and $+15 \mathrm{~V}$ having a common $0 \mathrm{~V}$ ground. This $0 \mathrm{~V}$-ground is connected to the source of the transistor to be driven. The gate driver is fed by an external $5 \mathrm{~V}$ DC voltage supply and the input signal, determining the frequency of the driver, is a block pulse of amplitude $5 \mathrm{~V}$, generated by a pulse generator. There are two galvanic isolations in the gate driver: one in the NMH0515DC-voltage supply and the second in the digital isolator ADUM1200CRZ. This isolator galvanically isolates its input from its output, but input and output voltages are basically the same. After the digital isolator, the signal may contain some noise. This noise can be reduced by a Schmitt trigger (SN74LVC1G17DBVR).

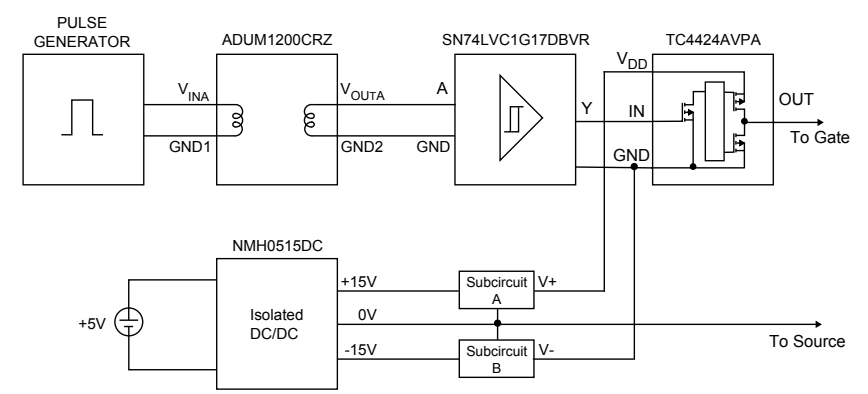

Fig. 1: Schematic of the basic principle of the first proposed gate-driver.

The driver can generate a positive gate-to-source voltage for switching the transistor on and a negative voltage for switching it off. The values of both voltages can be adjusted by using appropriate components in the two subcircuits $A$ and $B$, each containing emitter follower circuits. The subcircuit $A$ is shown in detail in Fig. 2(a).It converts the $+15 \mathrm{~V}$ from the DC/DC converter to a lower positive voltage which is determined by the Zener diode voltage $V_{Z}$. One can also use light-emitting diodes (LEDs) instead of the Zener diodes. In that case, the LEDs are reversely connected with respect to the Zener diodes.

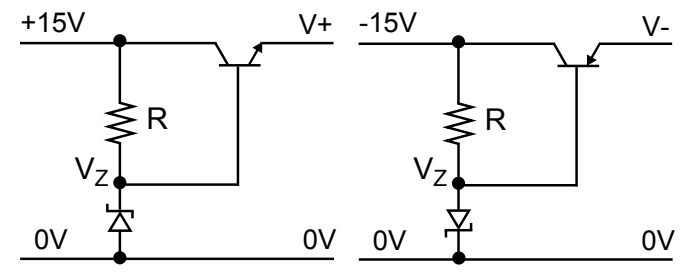

(a) Emitter follower $\mathrm{A}$

(b) Emitter follower B

Fig. 2: Emitter followers in subcircuits A and B.

The output voltages of the emitter followers $A$ and $B$ are respectively connected to the power and ground of the MOSFET driver TC4424AVPA. The negative voltage $V_{-}$ in Fig. 1 is connected to the ground (pin $G N D$ ) of the TC4424AVPA and the positive voltage $V_{+}$is connected to the pin $V_{D D}$. A more complete schematic of the gatedriver is given in Fig. 3

The proposed driver can easily be extended to a resonant version by putting a resonant tank-circuit between the gate of the transistor and the TC4424A. However, even using the conventional driver design, a frequency of multiple $\mathrm{MHz}$ can be reached (see further). Aside from its fast speed, another advantage of the first proposed gatedriver is that in fact, a system for power supply for a driver is presented above, which can also be used in a resonant driver circuit.

\section{B. Robustness}

If the pulse generator turns off for some reason, the MOSFET-driver TC4424AVPA receives a $0 \mathrm{~V}$ input signal 


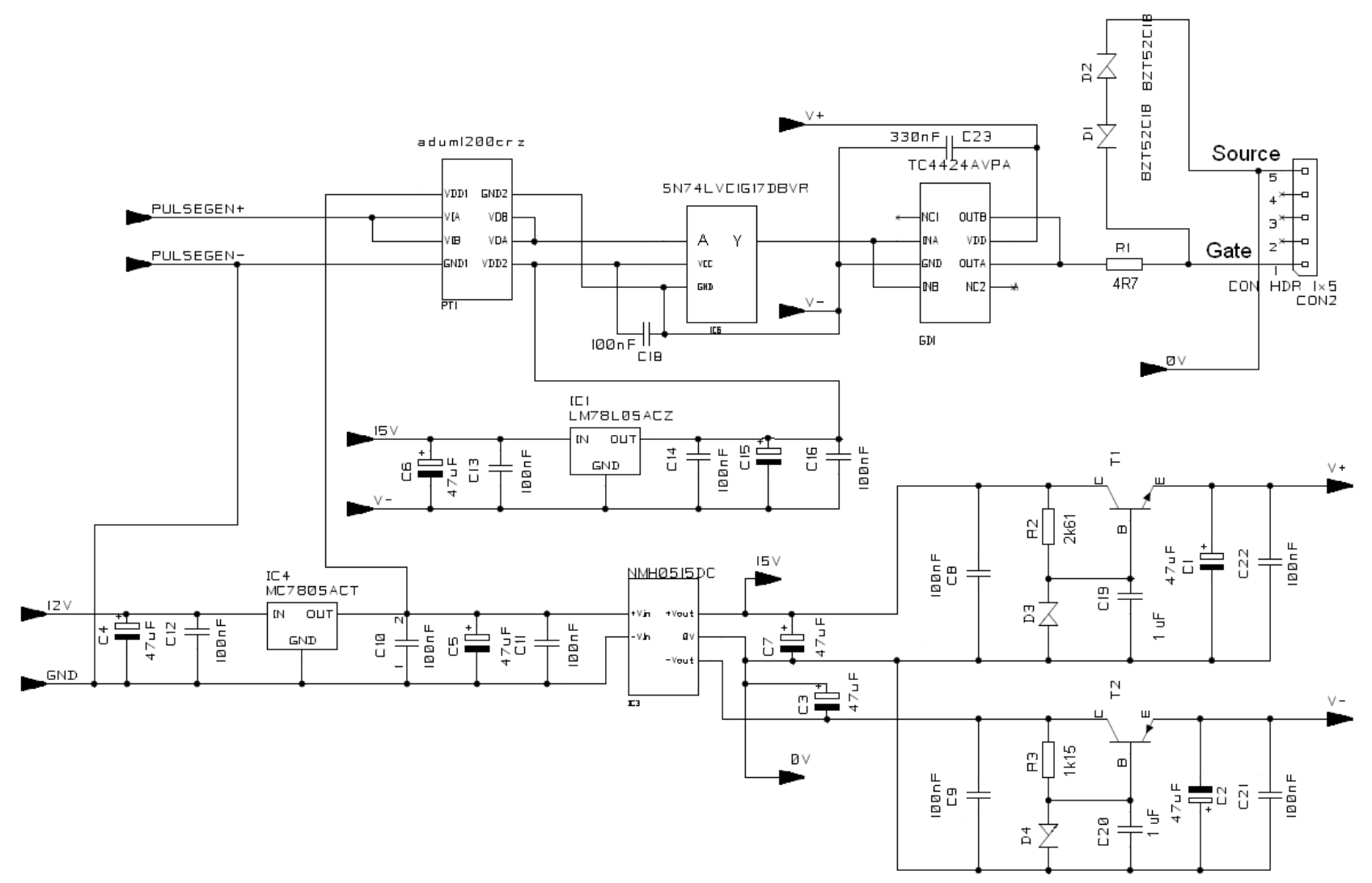

Fig. 3: Complete schematic of the first proposed gate-driver.

with respect to its ground. This means that at the output of the TC4424AVPA will be the negative voltage $V_{-}$with respect to the source of the transistor. Thus the gate-tosource voltage $V_{g s}$ across the GaN device is negative and the transistor will be turned off. This means that this gatedriver is well suited for normally-on components, such as the experimental GaN DHFET transistor of our concern [3].

\section{Performance}

Because the working depends on the use of emitterfollowers, the efficiency of the gate-driver is not so high. The voltage supply by means of two emitter-follower circuits represents one way of power supply. The gatedrivers in the next two sections have a different, more energy-efficient manner of power supply. However, when efficiency is of not a great importance, the driver presented above, still has many advantages. In the next few paragraphs some measurement results are presented which give an idea of the performance of the driver. The maximum frequency, from a thermal point of view, is first given. Next, the rise- and fall-times of the gate-tosource voltage are given when the driver is used to drive the transistor of our concern [3]. Finally, the fall-times are determined in function of the drain-to-source voltage, when the driver is operated on MOSFETs with a different input capacitance.

The maximum frequency is determined to be $6 \mathrm{MHz}$.
This is the frequency where the casing of the TC4424A is 55 degrees Celsius. The temperature is measured with a Peaktech 5110 thermometer. At $6 \mathrm{MHz}$, the case temperatures of the TIP120 and TIP125 are then 82 and 66 degrees Celsius, respectively. This is true if the driver is unloaded, and has to produce a gate-to-source voltage between 0.46 and $-8.24 \mathrm{~V}$. The rise and fall times are still quite low: 9.7 and $9.2 \mathrm{~ns}$.

The gate-driver was also used to drive the AlGaN/GaN/AlGaN DHFET [3] with a square wave voltage between 0.36 and $-8.16 \mathrm{~V}$ at $1 \mathrm{MHz}$. The rise and fall times are 13.3 and $9.5 \mathrm{~ns}$, respectively (Fig. 4).

The fall times $t_{\text {fall }}$ of the gate-to-source voltages (between 12 and $-3 \mathrm{~V}$ ) are examined for transistors with

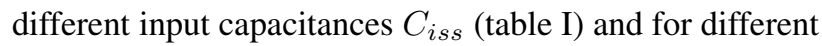
drain-to-source voltages $V_{d s}$. The values of the input capacitances are obtained from the transistor's datasheets and are the values at $V_{d s}=25 \mathrm{~V}, V_{g s}=0 \mathrm{~V}$ and at a frequency of $1 \mathrm{MHz}$, except for the IPW50R350CPtransistor where $V_{d s}=100 \mathrm{~V}$. The test setup is depicted in Fig. 5. The gate-to-source voltage is measured with a Tektronix P6250 differential probe (bandwidth $=500 \mathrm{MHz}$ ) and a Tektronix TDS5054 oscilloscope. The switching frequency is $10 \mathrm{kHz}$, and through the load flows a current of $3 \mathrm{~A}$. The resistance, placed at the gate of the transistor, is $4.75 \Omega$. A RC-snubber $(R=20 \Omega, C=2 \mathrm{nF})$ is used to diminish oscillations due to parasitics, at the drain-node, because this induces oscillations at the gate-node via the 


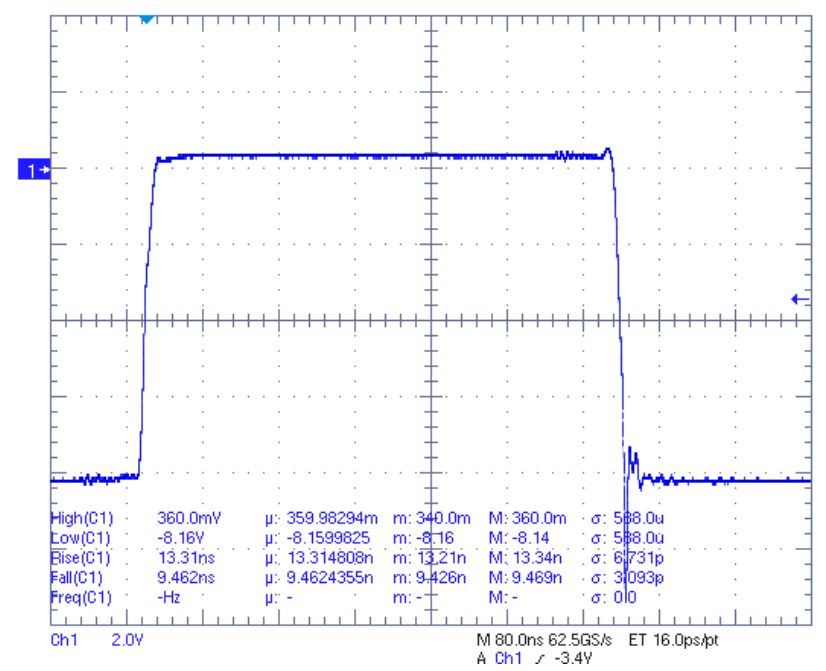

Fig. 4: Gate-to-source voltage of the driver when operated with the AlGaN/GaN/AlGaN DHFET [3] at $1 \mathrm{MHz}$.

internal drain-to-gate capacitance $C_{d g}$ of the MOSFET, making it harder to measure fall-times. Because in the rise time, there appear still oscillations, only the fall-time of the gate-to-source voltage is measured. The results are presented in Fig. 6 The fall-time increases for an increasing drain-voltage and for MOSFETs with a higher $C_{i s s}$, as is expected. It can be seen that the fall-time quickly saturates. Only the behavior of the MOSFET with $C_{i s s}=2600 \mathrm{pF}$ is slightly special because the measurements show that the fall-time is higher than that of the MOSFET with $C_{i s s}=3600 \mathrm{pF}$, for all drain-voltages. This figure shows the performance of the first proposed gate-driver, when operated on real MOSFETs at different voltage levels.

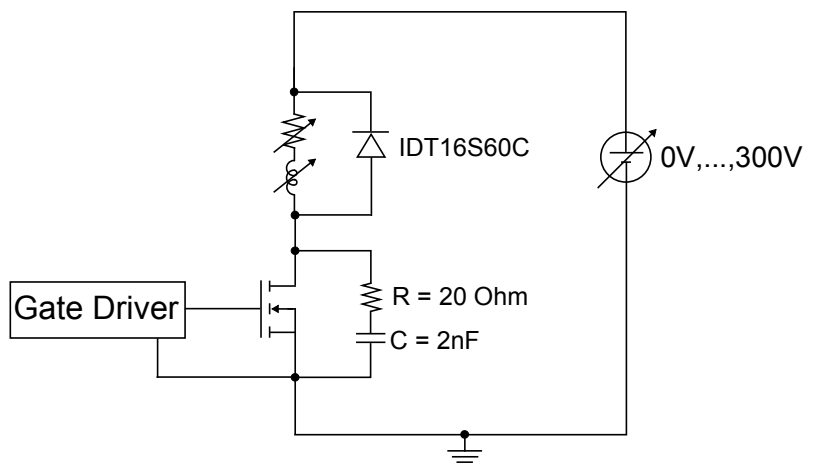

Fig. 5: Test setup to measure the fall-times of the gate-tosource voltage for different drain-voltages and MOSFETs with a different input capacitance.

D. Common-mode current due to the isolation capacitance of the DC-DC converter

The DC-DC-converter NMH0515DC contains a toroidal transformer having an isolation capacitance of $C_{i s o l}=33 \mathrm{pF}$ between its primary and secondary windings, according to its datasheet [12]. This is a rather large value. The output ground of the NMH0515DC

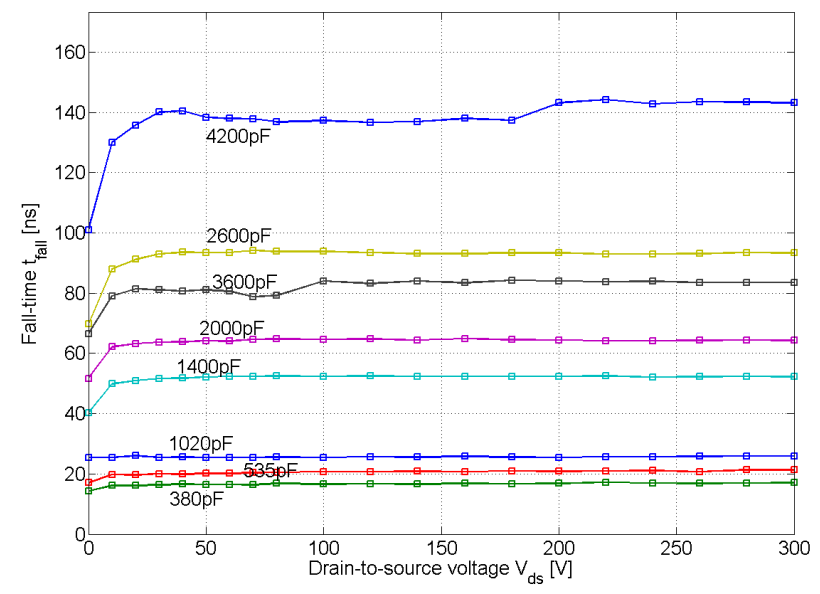

Fig. 6: Fall-time of the gate-to-source voltage for different drain-voltages and MOSFETs with a different input capacitance.

is always at the same potential as the source of the GaN-transistor. When the transistor is in its off-state, the output ground of the NMH0515DC is at $0 \mathrm{~V}$ with respect to the ground of the input voltage source of the buck circuit. When the transistor turns on, the output ground of the NMH0515DC is at $400 \mathrm{~V}$ with respect to the ground of the input voltage source of the power circuit. Due to the isolation capacitance in the DCDC-converter, there will flow a common-mode current through the NMH0515DC. This current is independent of the switching frequency but dependent on the rise and fall times of the switching voltage, and on the isolation voltage the converter has to withstand. The rise and fall times are in the order of $10 \mathrm{~ns}$. This results in a common-mode current with a peak value of:

$$
\begin{aligned}
i_{C M} & =C_{i s o l} \cdot \frac{d v}{d t} \\
& =33 \mathrm{pF} \cdot \frac{400 \mathrm{~V}}{10 \mathrm{~ns}}=1.32 \mathrm{~A}
\end{aligned}
$$

As every wire represents an impedance, a current flowing through it will induce a certain voltage drop across it. Due to this voltage drop, the input voltage of the NMH0515DC will not necessarily be a constant $5 \mathrm{~V}$ anymore. Because of this, the NMH0515DC, which requires a constant input voltage which must be, within

TABLE I: MOSFETs used for measuring the performance of the first proposed gate-driver, and their input capacitance

\begin{tabular}{|c|c|}
\hline MOSFET & $C_{i s s}[\mathrm{pF}]$ \\
\hline STP7NK30Z & 380 \\
STP7NK40Z & 535 \\
IPW50R350CP & 1020 \\
IRFP344PBF & 1400 \\
STW14NK50Z & 2000 \\
IRFP450PBF & 2600 \\
IRFP23N50LPBF & 3600 \\
IRFP460PBF & 4200 \\
\hline
\end{tabular}


strict limits, equal to $5 \mathrm{~V}$, can cease working properly. Normally, this problem can be circumvented by designing one's own DC-DC-converter where the primary and secondary windings are greatly spaced from each other. But because in this design, a commercially available DC-DCconverter is used with a rather large isolation capacitance, it is not so improbable that the problem here arises. The circuit therefore has been simulated in PSPICE as shown in Fig. 7. The GaN transistor switches $400 \mathrm{~V}$ at $1 \mathrm{MHz}$ and is driven with a voltage between 0.44 and $-7.9 \mathrm{~V}$. The LM7805C is a voltage regulator producing, in ideal conditions, a constant $5 \mathrm{~V}$ voltage for a higher (here, $12 \mathrm{~V}$ ) input voltage. The parasitic inductance $L 28 \| L 49$ between the grounds of NMH0515DC and LM7805C is about $(15.4 \cdot 8.47) /(15.4+8.47)=5.46 \mathrm{nH}[13]$. The output ground of the NMH0515DC is connected to the source of the switched $\mathrm{GaN}$ transistor. The transistors FDD3510HPCH and FDD3510HNCH form a half bridge circuit, representing the mosfet-driver IC TC4424AVPA. The half-bridge applies a $V_{g s}$ voltage between the gate and source of the DHFET-transistor, in the form of a square wave with values of $0.44 \mathrm{~V}$ and $-7.9 \mathrm{~V}$. The SPICE model used for simulating the NMH0515DC is:

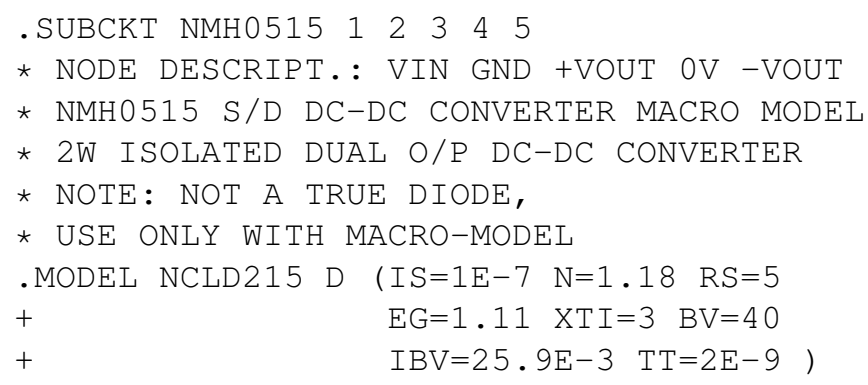

The common-mode current $i_{C M}$ (multiplied by 10 for clarity), gate-to-source voltage and input voltage of the DC-DC-converter are shown in Fig. 8. The simulation shows that despite of the common-mode current flowing through the isolation capacitance in the NMH0515DC, the input voltage of the NMH0515DC is within $5 \mathrm{~V}$ $\pm 0.07 \mathrm{~V}$. The simulation also shows that the peak value of the common-mode current is $0.45 \mathrm{~A}$, which is of the same order of magnitude as the calculated $1.32 \mathrm{~A}$. The allowed offset from $5 \mathrm{~V}$ for the NMH0515DC is $500 \mathrm{mV}$ [12], thus the DC-DC-converter still works according to specifications, as the simulation also shows.

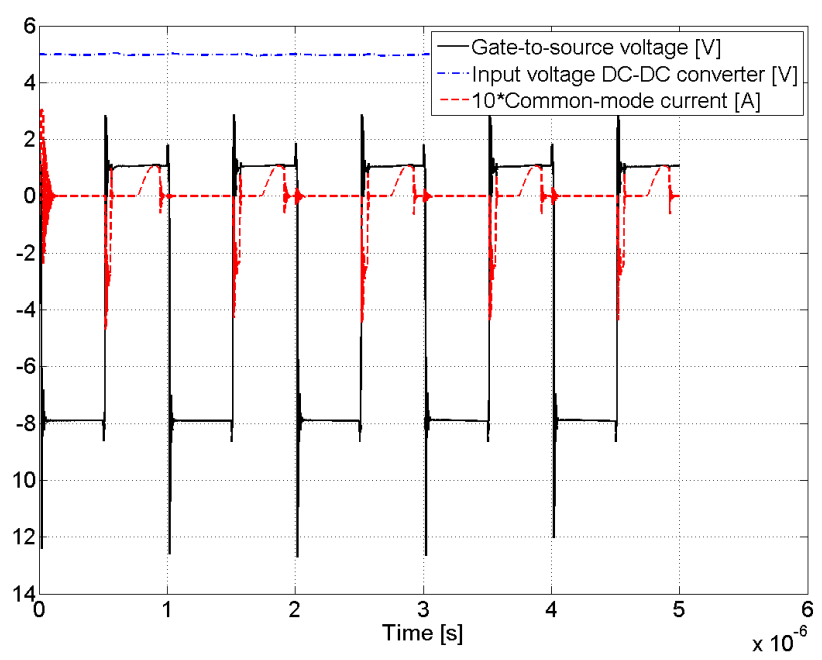

Fig. 8: Simulated currents and voltages in the NMH0515DC due to the presence of the common-mode isolation capacitance and parasitic inductances.

To confirm the simulation results, some practical tests are performed on the NMH0515DC with the circuit from Fig. 9. The HCPL3180 is an optocoupler that serves for the galvanic isolation of the gate-driver. The operation of the gate-driver has been assessed for a drain-to-source voltage $V_{d s}=300 \mathrm{~V}$ and a switching frequency $f=$ $100 \mathrm{kHz}$. The IRFP460A MOSFET is driven at a $0 / 15 \mathrm{~V}$ gate-to-source voltage. The load consists of a $267 \Omega$ resistor. A freewheeling diode (15ETH06) is used in the circuit. The gate-to-source voltage is measured to see whether it is still correctly generated. A Tektronix P5200 differential voltage probe (bandwidth $=25 \mathrm{MHz}$ ) and the oscilloscope TDS5054 $(500 \mathrm{MHz})$ of Tektronix were used. Because of the presence of parasitic inductances and the fact that there is a common-mode current flowing through them, points $B$ and $C$ (Fig. 9) are not on the same potential. Thus, there may not be $5 \mathrm{~V}$ between the points $A$ and $B$, at the input of the NMH0515DC. The gate-to-source-voltage is shown in Fig. 10. This test shows that even though there is a common-mode current due to the isolation capacitance, the gate-driver still generates correctly a $0 / 15 \mathrm{~V}$-square wave pulse. Therefore, it is proven that the NMH0515DC works correctly, even with the presence of a parasitic isolation capacitance. 


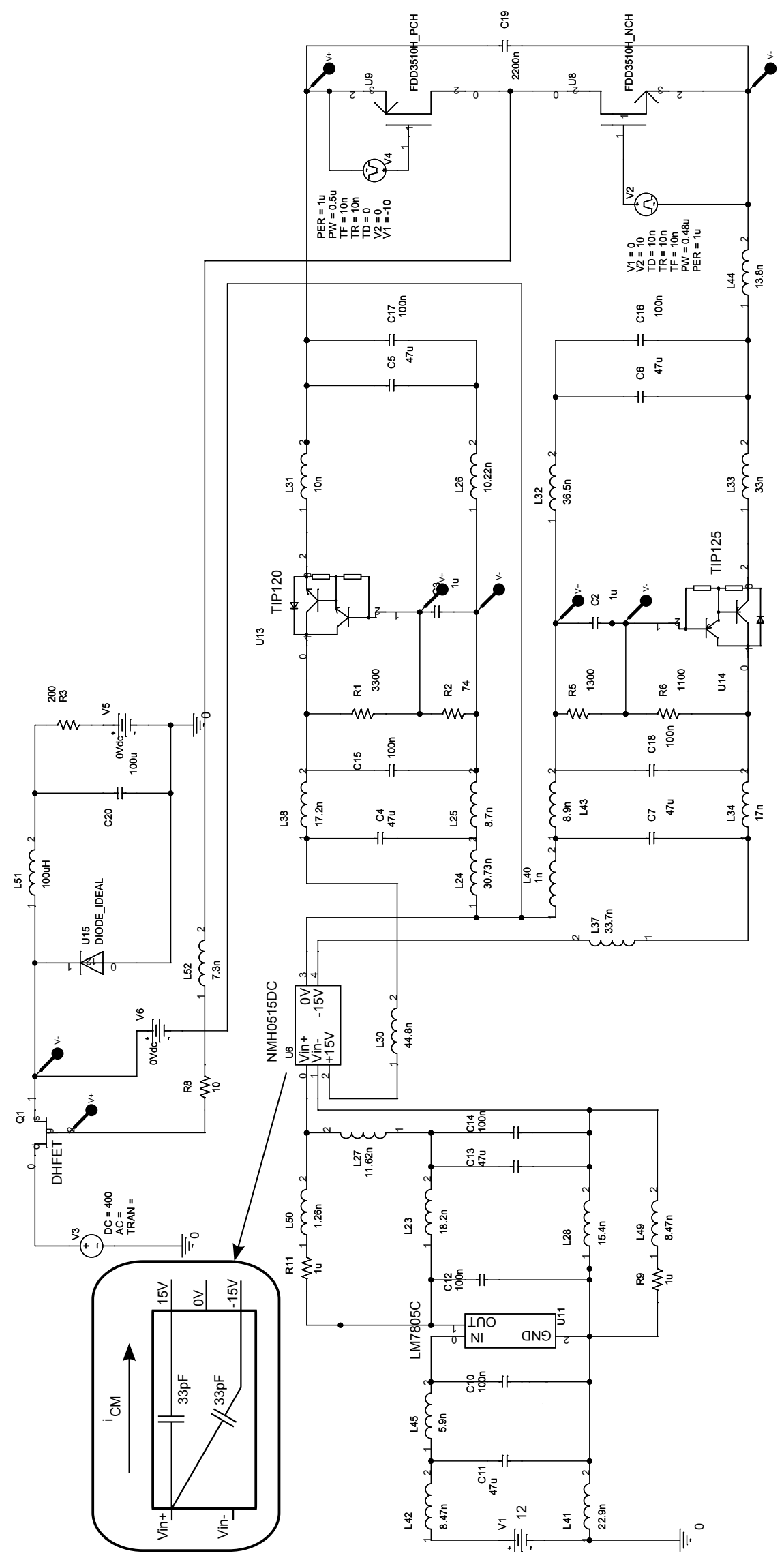

Fig. 7: PSPICE simulation of the presence of the isolation capacitance in the NMH0515DC. 


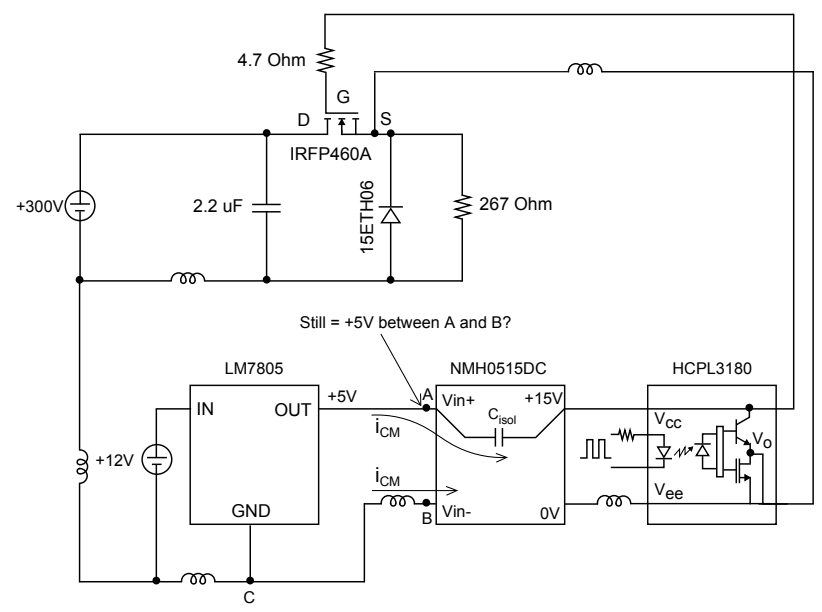

Fig. 9: Practical circuit for testing the problem of the common-mode current through the NMH0515DC.

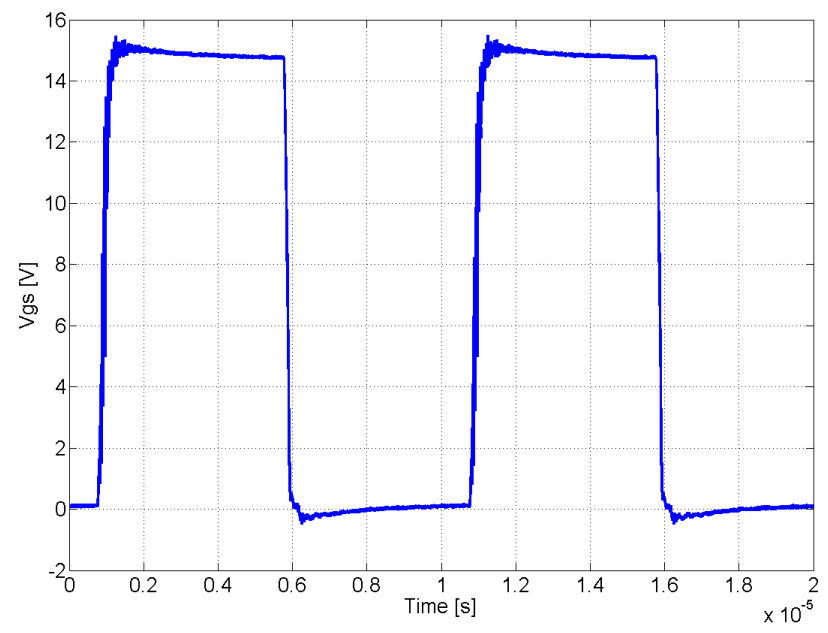

Fig. 10: Gate-to-source voltage in the test circuit of Fig. 9.

III. SECOND PROPOSED GATE-DRIVER, WHERE A DC-DC-CONVERTER IS PLACED IN SERIES WITH THE GATE

\section{A. Topology}

The circuit, proposed in Fig. 11, is based on a conventional 9 A high speed MOSFET drive IC (TC4422A). This IC has a peak output current of $10 \mathrm{~A}$, a wide input supply voltage $\left(V_{d d}\right)$ operating range of $4.5 \mathrm{~V}-18 \mathrm{~V}$, fast rise and fall times of $15 \mathrm{~ns}$ with $4700 \mathrm{pF}$ load and a low output impedance of $1.2 \Omega$ (see datasheet [14]), making it very suitable for driving the largest transistors under very high frequencies. The output voltage $\left(V_{A}\right)$ of this drive IC switches between zero and $V_{d d}$, based on a pulse signal $\left(V_{\text {pulse }}\right)$. This signal indirectly comes from a Field-Programmable Gate Array (FPGA) and is electrically isolated first. The input supply voltage $\left(V_{d d}\right)$ comes from rectifier, fed by the secondary winding of a transformer. A simple isolated DC-DC-converter is put in series with the output of the drive IC, providing a negative offset $\left(V_{o f f}\right)$ to $V_{A}$. By doing this, a fully controllable

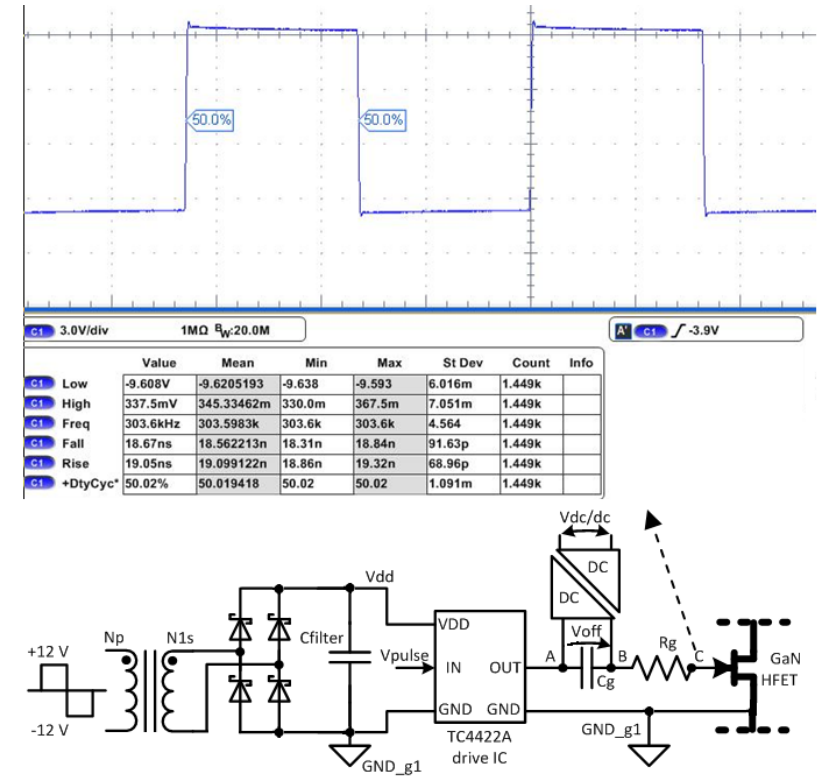

Fig. 11: Principle scheme of the second proposed gatedrive circuit. (Inset) Waveform at the gate (point $\mathrm{C}$ ), showing a voltage range of $-9.608 \mathrm{~V} / 0.337 \mathrm{~V}$.

drive-voltage range $\left(V_{B}\right)$ is obtained:

$$
\begin{gathered}
V_{A}=\left[0 / V_{d d}\right] \\
V_{B}=V_{A}-V_{o f f} \\
\Longrightarrow V_{B}=\left[0-V_{o f f} / V_{d d}-V_{o f f}\right]
\end{gathered}
$$

where $V_{\text {off }}$ is controlled by adjusting the input voltage $\left(V_{D C / D C}\right)$ of the DC-DC-converter and $V_{d d}$ by adjusting the winding ratio of the transformer. Even positive voltage ranges are possible by simply removing the offset voltage $V_{o f f}$, giving the possibility to test conventional silicon power transistors and enabling comparison of different types of transistors (AlGaN/GaN/AlGaN DHFET, CoolMOS ${ }^{\mathrm{TM}}$, MOSFET,...). A capacitor $\left(C_{g}\right)$ is placed in parallel with the DC/DC converter to short circuit the dynamic current pulses. The gate resistor $\left(R_{g}\right)$ determines the switching speed and can easily be replaced to investigate its influence on the behavior of the tested devices.

\section{B. Robustness}

This second driver is also robust. That is, if the pulse signal from the FPGA or pulse generator falls away, a negative gate-to-source voltage equal to $-V_{\text {off } f}$ is applied.

\section{Performance}

The inset of Fig. 11 shows the gate voltage waveform, measured directly at the gate (point $\mathrm{C}$ ) of the AlGaN/GaN/AlGaN DHFET. The pulsed voltage has a frequency of $303.6 \mathrm{kHz}$, a duty cycle of $50.02 \%$ and a range of $-9.608 \mathrm{~V} / 0.337 \mathrm{~V}$. The rise and fall times are 19.05 and $18.67 \mathrm{~ns}$, respectively. These fast switching times are the result of a good driver design, combined with the very low gate capacitance and resistance of the AlGaN/GaN/AlGaN DHFETs. This results in reduced 


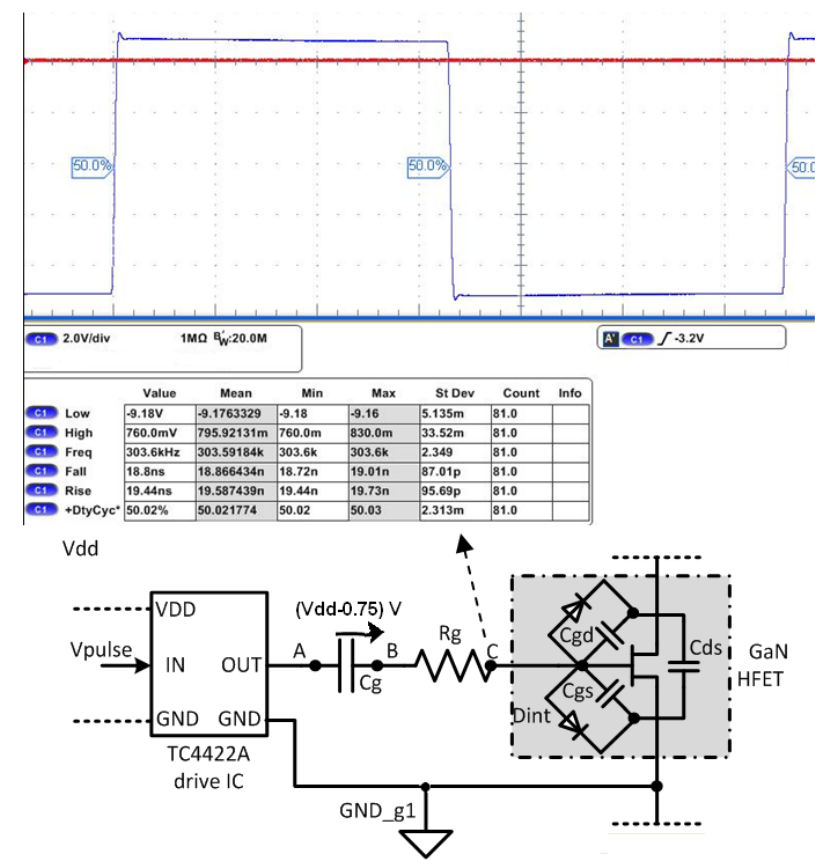

Fig. 12: Principle scheme of an alternative gate-drive circuit, making use of the internal gate-drain diode $\left(D_{\text {int }}\right)$ of the AlGaN/GaN/AlGaN DHFETs. (Inset) Waveform at the gate (point $\mathrm{C}$ ), showing a voltage range of $-9.18 \mathrm{~V} /$ $0.76 \mathrm{~V}$

switching losses, enabling higher switching frequencies in power electronic converters.

\section{THIRD PROPOSED GATE-DRIVER, MAKING USE OF THE INTERNAL GATE-TO-SOURCE DIODE OF THE} DHFET

\section{A. Topology}

Fig. 12 Presents an alternative way to drive the AlGaN/GaN/AlGaN DHFET, using its internal gate-source diode $\left(D_{\text {int }}\right)$. Here the DC-DC-converter from the above proposed gate-drive circuit is simply removed. When $V_{A}$ rises to $V_{d d}, D_{\text {int }}$ starts to conduct for a moment and $V_{B}$ equals the diode voltage drop $V_{d}$ (typically $0.75 \mathrm{~V}$ ). When $V_{A}$ drops to zero, the charged capacitor pulls $V_{B}$ to zero minus the capacitor voltage, being $V_{d d}-V_{d}$. This gives a voltage range $V_{B}$ of:

$$
V_{B}=\left[-\left(V_{d d}-V_{d}\right) / V_{d}\right]
$$

\section{B. Robustness}

This third driver is, however, not robust. That is, if the pulse signal from the FPGA or pulse generator falls away, and capacitor $C_{g}$ is discharged after a while, zero volt is applied between gate and source instead of a negative voltage.

\section{Performance}

This drive circuit was tested and gives similar results as the previous one, making it suitable for use in an AlGaN/GaN/AlGaN-based converter. For testing, it does not offer the same flexibility as the previously mentioned circuit. The inset of Fig. 12 shows the waveform of the gate voltage $V_{C}$. It can be seen that the on-state gate voltage is $0.76 \mathrm{~V}$ as predicted.

\section{CONCLUSION}

This paper presents three different gate-drivers which have been used to drive a new normally-on AlGaN/GaN/AlGaN-DHFET transistor. For each driver, the topology and working principle is discussed. The drivers are (1) galvanically isolated and are, except for the third driver, (2) robust (fail-proof). Furthermore (3), it is possible to easily modify the driver circuit to generate different gate-to-source voltage levels. Finally (4), it is possible to construct the drivers with standard available electronic components. In isolated drivers, if the isolation capacitance has a high value, a common-mode current flows which interferes with the driver. This issue was also examined but showed to be of no concern for the presented drivers.

\section{REFERENCES}

[1] B. Ozpineci, L. M. Tolbert, S. K. Islam, and M. Chinthavali, "Comparison of wide bandgap semiconductors for power applications," in Proc. of 10th European Conference on Power Electronics and Applications, Toulouse, France, Sep. 2003.

[2] K. Takahashi, A. Yoshikawa, and A. Sandhu, Wide Bandgap Semiconductors - Fundamental Properties and Modern Photonic and Electronic Devices. Berlin: Springer, 2007.

[3] D. Visalli, M. V. Hove, J. Derluyn, S. Degroote, M. Leys, K. Cheng, M. Germain, and G. Borghs, "Algan/gan/algan double heterostructures on silicon substrates for high breakdown voltage field-effect transistors with low on-resistance," Jpn. J. Appl. Phys., vol. 48, Apr. 2009, 04C101.

[4] D. Maksimovic, "A mos gate drive with resonant transitions," in IEEE App. Power Electron. Conf., 1991, p. 527532.

[5] Y. Chen, F. Lee, L. Amoroso, and H. Wu, "A resonant mosfet gate driver with complete energy recovery," in Proc. IEEE Power Electronics and Motion Control Conference, vol. 1, 2000, p. 402 406.

[6] I. D. Vries, "A resonant power mosfet/igbt gate driver," in 17th Annual IEEE Applied Power Electronics Conference and Exposition, vol. 1, Mar. 10-14, 2002, pp. 179 - 185.

[7] R. Tzeng and C. Chen, "A low-consumption regulated gate driver for power mosfet," IEEE Transactions on Power Electronics, vol. 24, no. 2, pp. 532 - 539, Feb. 2009.

[8] T. López, G. Sauerlaender, T. Duerbaum, and T. Tolle, "A detailed analysis of a resonant gate driver for pwm applications," in Proc. IEEE App. Power Electron. Conf., vol. 2, 2003, p. 873878.

[9] K. Hwu and Y. Yau, "Application-oriented low-side gate drivers," IEEE Transactions on Industry Applications, vol. 45, no. 5, pp. 1742 - 1753, Sep./Oct. 2009.

[10] L. Li, M. Yu, X. Xiaogao, Z. Chen, Y. Wei, and Q. Zhaoming, "A new resonant gate driver for low voltage synchronous buck converter based on topologies optimization," in 23rd Annual IEEE Applied Power Electronics Conference and Exposition, Feb. 2428, 2008, pp. $1067-1072$.

[11] H. Wang and F. Wang, "A self-powered resonant gate driver for high power mosfet modules," in IEEE App. Power Electron. Conf., 2006, p. 183188.

[12] [Online]. Available: http://www.murata-ps.com

[13] I. J. Bahl and R. Garg, "Simple and accurate formulas for microstrip with finite strip thickness," Proc. IEEE, vol. 65, no. 11 , pp. 1611-1612, Nov. 1977.

[14] [Online]. Available: http://www.microchip.com 